\title{
BMJ Open Health-related quality of life among patients with hypertension: population- based survey using EQ-5D-5L in Hong Kong SAR, China
}

\author{
Eliza Lai Yi Wong, Richard Huan Xu (D) , Annie Wai Ling Cheung
}

To cite: Wong ELY, Xu RH, Cheung AWL. Healthrelated quality of life among patients with hypertension: population-based survey using EQ-5D-5L in Hong Kong SAR, China. BMJ Open 2019;9:e032544. doi:10.1136/ bmjopen-2019-032544

- Prepublication history for this paper is available online. To view these files, please visit the journal online (http://dx.doi. org/10.1136/bmjopen-2019032544).

Received 25 June 2019 Revised 26 August 2019 Accepted 04 September 2019

Check for updates

(C) Author(s) (or their employer(s)) 2019. Re-use permitted under CC BY-NC. No commercial re-use. See rights and permissions. Published by BMJ.

Jockey Club School of Public Health and Primary Care, The Chinese University of Hong Kong, Hong Kong SAR, China

Correspondence to Dr Richard Huan Xu; richardhxu@cuhk.edu.hk

\section{ABSTRACT}

Objectives To evaluate the effect of hypertension and related comorbidities on the health-related quality of life (HRQOL) using EuroQol 5-dimensions instrument with fivelevel scale (EQ-5D-5L) Hong Kong (HK) version.

Design Data were analysed by a secondary data analysis based on a cross-sectional study assessing experience on public specialist outpatient service.

Setting A representative sample was recruited from 26 specialist outpatient clinics in HK.

Participants A total of 4528 patients with hypertension aged 18 or above who responded to the survey. Intervention EQ-5D-5L HK was applied to assess the $\mathrm{HRQOL}$. The five-dimension descriptive system and the utility index of EQ-5D-5L were treated as the dependent variable in the current study. Regression modelling was applied to estimate the effect of hypertension and related comorbidities on health-related quality of life.

Results More respondents were women (53.9\%), aged $\geq 65$ years old $(60.1 \%)$, and with primary educational attainment or below (52.3\%). A total of 1466 respondents $(32.4 \%)$ also reported suffering from diabetes, heart disease $(20.8 \%)$, vision problem $(1.7 \%)$ and cancer $(1.5 \%)$. In the ordinal least squares model, utility decreased most when patients reported having physical disability associated with hypertension (beta $=-0.395, \mathrm{SE}=0.047$ ), and $0.128,0.064,0.05$ and 0.048 for mental problem, cancer, vision problem and heart problem. In the Tobit model, the utility reduced most for comorbidity of physical disability, and then mental problem, cancer, vision problem, heart problem and diabetes. For first part of two-part model, respondents coliving with mental problem were $10 \%$ less likely to report a full health. For the second part of two-part model, the respondents with physical disability had 0.294 lower in utility.

Conclusions Respondents with hypertension reported a Iow EQ-5D utility score. Respondents were women, with a high education, fully employed and living with families reported better HRQoL. There is a significant effect of comorbidity influence on the decreased HRQOL, particularly those with physical disability and mental problem.

\section{INTRODUCTION}

Hypertension is a chronic condition in which blood pressure in the arteries is persistently elevated. At present, hypertension is a global

\section{Strengths and limitations of this study}

- This is a large population-based study to assess the association of hypertension and associated comorbidities on the preference-based health-related quality of life (HRQDL) in Hong Kong, China.

- Three different regression models were applied to estimate the effect of hypertension on HRQoL.

- The patients who lived in institutions (eg, hospitals, old age homes or nursing care homes) and persons living aboard vessels were not involved in the study. Thus, further studies are suggested to enrich the findings in the local population.

public health issue that dramatically increases the risk of heart disease, stroke, kidney failure and physical disability. ${ }^{1}$ WHO indicated that approximately $40 \%$ of adults aged $\geq 25$ years have been diagnosed with hypertension worldwide, and the number increased from 600 million in 1980 to 1 billion in $2008 .^{2}$ In Hong Kong (HK), hypertension is a silent killer. The Department of Health reported that nearly $30 \%$ of people aged $\geq 15$ years live with hypertension. The prevalence increases steadily from $4.5 \%$ among people aged $15-24$ years to $64.8 \%$ among those aged $65-84$ years. However, only $13.2 \%$ of patients with hypertension are aware of their condition. ${ }^{3}$

Recently, instead of clinical outcomes, such as morbidity, the health-related quality of life (HRQoL) of patients has been increasingly studied in hypertensive research. ${ }^{4}$ Living with hypertension can influence a patient's physical health, psychological state, independence level and family and social relationships; it ultimately leads to decreased HRQoL. ${ }^{5-8}$ Comorbidity associated with hypertension is another independent factor that influences HRQoL. ${ }^{910}$ Pinto indicated that in hypertension, sociodemographic characteristics, rather than disease characteristics, may play an important role in assessing the HRQoL 
of patients. ${ }^{11}$ However, a lot of current disagreements in the findings of hypertensive studies are due to the use of different tools for measuring HRQoL. ${ }^{12}$ Debates on which between profile-based instruments and preference-based instruments or between generic measures and condition-specific measures should be used have persisted. In recent decades, EuroQol 5-dimensions instrument (EQ-5D) has been recommended as one of the most popular generic preference-based instrument to assess patient HRQoL worldwide. ${ }^{13}$ In particular, its new version of EQ-5D with five-level scale, namely EQ-5D-5L, has been highly suggested due to its ability to reduce the ceiling effect. The good validity and reliability of EQ-5D-5L in certain chronic conditions have been demonstrated. ${ }^{14-17}$ However, the relative effects of hypertension and related comorbidity on HRQoL using EQ-5D-5L in HK have been rarely explored.

This study was proposed to provide empirical evidence to connect hypertension together with related comorbidity and HRQoL and also encourage clinical professionals and policy-makers to list HRQoL improvement as one of the most important therapeutic and political objectives in the healthcare of patients with hypertension. Therefore, this study aimed: (1) to evaluate how hypertension and related comorbidities affect HRQoL by using EQ-5D-5L HK; (2) to identify how sociodemographic characteristics affect hypertension-HRQoL relationship and (3) to explore how different regression models account for the distributional features of EQ-5D utility.

\section{METHOD}

\section{Data collection}

We used a secondary data from a territory-wide cross-sectional patient experience survey on specialist outpatient clinic services (SOPCs) in HK. ${ }^{18}$ The survey was conducted among the patients who attended at the 26 selected public SOPCs under hospital authority. Recruits must be aged 18 or above and able to speak and understand Cantonese. Patients who were day cases or day surveys or those from paediatric, hospice, psychiatric, dental, anaesthesiology, pathology or nurse-led or multispecialty outpatient clinics were excluded. The respondents were requested to answer a structured questionnaire over the phone within 2 weeks after their attendance at SOPCs. Besides the evaluative questions on patient experience, the respondents were also asked to report their health status using EQ-5D-5L HK (descriptive system) and self-reported long-standing conditions such as their physical status and whether had chronic diseases. In that survey, hypertension was defined as persistent systolic blood pressure (SBP) of $\geq 140 \mathrm{~mm}$ $\mathrm{Hg}$ or diastolic blood pressure of $\geq 90 \mathrm{~mm} \mathrm{Hg}$ based on the criteria suggested by the Centre for Health Protection Hong Kong (www.chp.gov.hk/en/index.html). A total of 13966 patients responded the patient experience survey and 4528 of them reported living with hypertension. The respondents who reported having hypertension and their information was elicited and analysed in this study.

\section{Comorbidity}

In the count method, comorbidity was defined as two chronic conditions that occur simultaneously. ${ }^{19}$ Nine chronic conditions, namely, vision problem, hearing problem, physical disability, learning problem, mental problem, heart disease, hypertension, diabetes and cancer were identified in the patients with hypertension. However, no one reported having a learning problem together with hypertension. Thus, seven comorbidities, namely (1) hypertension and heart disease; (2) hypertension and diabetes; (3) hypertension and cancer; (4) hypertension and vision problem; (5) hypertension and hearing problem; (6) hypertension and physical disability and (7) hypertension and mental problem, were included in the analysis to evaluate the effect of comorbidities associated with hypertension on HRQoL in this study.

\section{Study instrument-EQ-5D-5L}

EQ-5D-5L is a simple, generic and standardised instrument for assessing patient health states for clinical and economic appraisal. ${ }^{13}$ EQ-5D-5L essentially consists of two parts: the descriptive system and EQ Visual Analogue Scale (EQ-VAS). Descriptive system has five dimensions: mobility (MO), self-care (SC), usual activities (UA), pain/ discomfort (PD) and anxiety/depression (AD). Each dimension has five levels (no problem, slight problems, moderate problems, severe problems and extreme problems). The combinations of dimensions and levels can be used to define the different health states of HRQoL (total of 3125 health states). EQ-5D-5L is the new version of the original EQ-5D-3L (three levels for each dimension), and it has been confirmed to exhibit improved ability to control the ceiling effect. The EQ-5D-5L HK version was developed and validated in HK cultural settings following the latest international protocol in 2017, which allowed the evaluation of people's HRQoL by considering their perceptions in the HK context of culture and value systems. ${ }^{20}$ All health states defined by EQ-5D-5L can be converted into a single summary index called utility. Utility can support the calculation for quality-adjusted life years as a reference for the health economic evaluations of healthcare policies or clinical interventions. The calculation of utility was based on an HK value set. The basic formula is utility $=1-\beta_{1} * \mathrm{MO}-\beta_{2}{ }^{*} \mathrm{SC}-\beta_{3}{ }^{*} \mathrm{UA}-\beta_{4}{ }^{*} \mathrm{PD}$ $-\beta_{5}{ }^{*} \mathrm{AD} .{ }^{20}$ The value of $\beta$ depends on the levels selected by the respondent at each dimension. The utility value ranges from -1 to 1 (full health). For the HK general population, it ranged from -0.86 ( 0 indicates death, $<0$ indicates worse than death) to 1.0. The mean utility was 0.92 . However, the design of the patient experience questionnaire on SOPC service only includes the five questions of the EQ-5D descriptive system and not includes the EQ-VAS. Therefore, we only applied our local derived algorithm to get the health-related utility index based on the data collected from EQ-5D descriptive system for the analysis in this manuscript. 


\section{Statistical analysis}

Descriptive statistics were used to summarise respondent characteristics and EQ-5D utility data. All demographic and socioeconomic characteristic indicators were grouped for analysis. Age and educational attainment were categorised into three groups, respectively. Living status and employment status were used as proxy questions to reflect the respondents' economic situation. Given that EQ-5D utility was non-normally distributed (Shapiro-Wilk test, $\mathrm{p}<0.05$ ), differences in EQ-5D utility among sociodemographic subgroups were assessed using a bootstrap version of the heteroscedastic one-way analysis of variance for trimmed means. This is a robust statistical method introduced by Wilcox. ${ }^{21}$

For regression models, considering that the ceiling effect of EQ-5D utility in this study was $46.5 \%$, we used different models to estimate the effect of hypertension and associated comorbidities on HRQoL adjusted by sociodemographic characteristics. The first model was the ordinal least squares (OLS) model. OLS is a traditional and classical method for regression analysis. However, EQ-5D utility data were censored (range between -0.8637 and 1 in current study), which posed a considerable challenge for using the OLS model in the analysis and consequently, several biassed estimations might be made. The second model was the Tobit regression model. This model is a censored model and was designed to estimate linear relationships among variables when either left or right censoring occurs in the dependent variable. ${ }^{22}$ Considering that nearly half of the respondents reported full health (utility=1.0) in this study, a two-part model (logistic regression and truncated OLS model) was also adopted to estimate the effect of hypertension on HRQoL. ${ }^{23}$ Logistic regression model was used to predict the probability of respondents reporting full health $(0$ and 1 , where 0 indicates non-full health and 1 indicates full health). Truncated OLS model was used to predict the probability of the respondents reporting non-full health (utility $\neq 1.0$ ). The results of the two types of models were combined to obtain an overall value. ${ }^{24}$

Two submodels were used for each of the three regression models. The first submodel estimated the impact of hypertension on HRQoL adjusted by sociodemographic characteristics. The second submodel estimated the effect of comorbidity associated with hypertension on HRQoL adjusted by sociodemographic characteristics (total of six models). To assess the fit of the models, we applied a series of criteria based on the suggestions of Wu et al. ${ }^{22}$ The root mean square error (RMSE) and sigma were used to estimate the accuracy of the models. RMSE indicated the fit of the OLS model, and sigma was used to indicate the fit of the Tobit model and the second part of the two-part model. When comparing the values of RMSE or sigma, a small value represents a small error, and consequently, a good model. Moreover, the Akaike information criterion (AIC) and the Bayesian information criterion (BIC) were used to evaluate the overall fit of the models. Pairwise deletion was performed to resolve missing values. Data were analysed using $\mathrm{R}$ (R Foundation, Austria), and statistical significance was set as $\mathrm{p}<0.05$.

\section{Patient and public involvement}

No patients were involved in the design of the study. They were recruited from 26 SOPCs in HK and informed of the details of the study, including its purpose, the process and the potential results, as well as their rights and responsibilities. We also assured the participants that all collected information would be kept confidential and anonymous. We gratefully acknowledged the contributions of all participants.

\section{RESULTS}

Table 1 indicates more respondents were women $(53.9 \%)$, aged $\geq 65$ years old $(60.1 \%)$, and with primary educational attainment or below (52.3\%). Approximately $93.2 \%$ of the respondents indicated living with family or others, and $57.6 \%$ were retired. A total of 1466 respondents $(32.4 \%)$ also reported cosuffering from diabetes, heart disease $(20.8 \%)$, vision problem $(1.7 \%)$ and cancer $(1.5 \%)$. Highly educated young male respondents living with family/others or fully employed reported having high EQ-5D utility. Moreover, respondents reported within hypertension scored significantly lower EQ-5D utility than respondents without it in the SOPC survey.

Figure 1 shows the percentage of respondents who reported problems in the five dimensions of EQ-5D for men and women separately. More women reported full health than men on SC (45\% vs $42 \%)$ and $\mathrm{AD}(42 \%$ vs $38 \%)$. By contrast, more men (28\%) than women (25\%) reported full health on PD. Nearly $20 \%$ of women reported having slight problems on $\mathrm{PD}$, followed by $\mathrm{MO}$ $(20 \%)$, UA (20\%) and AD (20\%). Figure 2 presents the distribution of EQ-5D utility for overall, man and woman separately. EQ-5D utility was heavily skewed, with more men $(45 \%)$ reporting full health than women $(42 \%)$.

Table 2 presents the results of OLS, Tobit and the two-part regression models. Although directly comparing the fit of the two-part model with the OLS or Tobit model is impossible, a comparison can be made based on the values of RMSE and sigma. In this study, the RMSE value of the OLS model was lower than the sigma value of the second part of the two-part model and the Tobit model. This result indicated that the OLS model was more accurate than the Tobit and two-part models. On the basis of the AIC and BIC values, the OLS model achieved the best performance, followed by the two-part model, and the Tobit model ranked last.

In estimating the effects of hypertension and associated comorbidities on EQ-5D utility, the OLS and Tobit models presented similar patterns. For the OLS and Tobit models, the coefficient of sociodemographic characteristics barely changed with and without considering comorbidities. This finding indicated that the relationship between sociodemographic characteristics and self-reported chronic conditions with the predicted EQ-5D utility was 
Table 1 Sociodemographic characteristics of the respondents

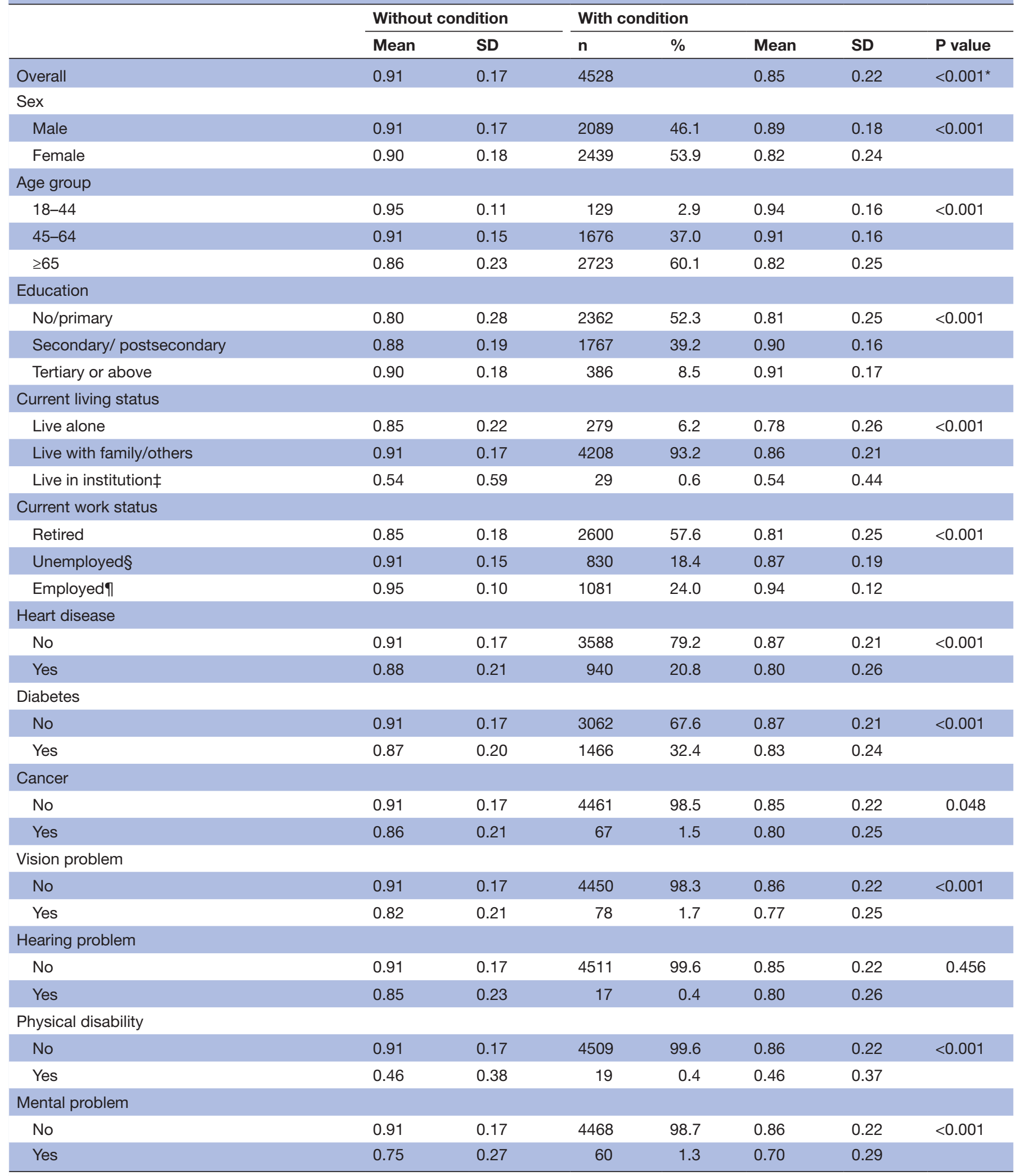

${ }^{*} \mathrm{P}$ value indicated that there is a significant difference of EuroQol 5-dimensions utility between with hypertension and without hypertension. $\dagger \mathrm{No}=$ No formal education/kindergarten; secondary included lower secondary=F0.1-F.3 and upper secondary=F0.4-F.5; postsecondary included postsecondary and matriculation=F0.6-F.7. c 53 refused to answer, the total response is 13913.

łInclude Convalescent Hospital/Rehabilitation Hospital/Hospital, and old age home.

§Unemployment included unemployed, home maker and full-time student.

ПEmployment included full-time worker and part-time worker. 


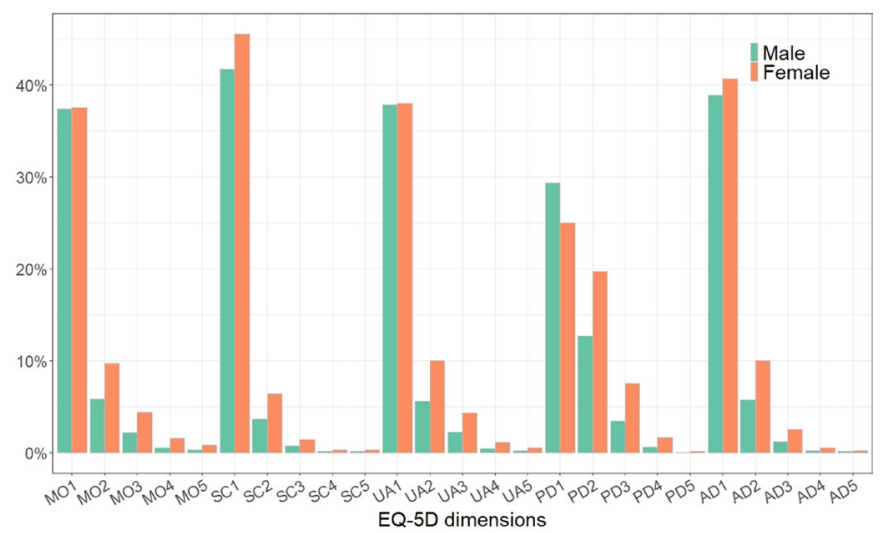

Figure 1 Distribution of EuroQol 5-dimensions by sex. AD, anxiety/depression; MO, mobility; PD, pain/discomfort; SC, self-care; UA, usual activities.

independent of each other. Moreover, the AIC and BIC values suggested that the comorbidity model performed better than that without the comorbidity model. Thus, it supported our hypothesis that comorbidity should be considered in the models.

In the OLS model, utility decreased by 0.067 when the patients were women. For fully employed and unemployed respondents, utility increased by 0.6 and 0.56 , respectively, compared with that for retired respondents. For comorbidity, utility decreased most when patients reported having a physical disability together with hypertension (beta $=-0.395, \mathrm{SE}=0.047$ ). Moreover, utility decreased by $0.128,0.064,0.05$ and 0.048 for patients with hypertension with mental problem, cancer, vision problem and heart problem, respectively. The comorbidity of diabetes with hypertension exerted the least effect on HRQoL than the other comorbidities. Compared with the OLS model, the Tobit model resulted in a higher reduction in EQ-5D utility. Women (beta $=-0.109, S E=0.011$ ) in the Tobit model were associated with substantially lower utility than men. The utility for respondents aged $\geq 65$ years decreased by 0.013 compared with those for respondents younger than 44 years. For respondents with tertiary and secondary/postsecondary educational attainment, utility increased by 0.95 and 0.82 , respectively. For comorbidity, we identified findings similar to those in the OLS model. Utility was reduced most for the comorbidity of physical disability (beta $=-0.469, \mathrm{SE}=0.071$ ), and utility decreased by $0.18,0.097,0.086,0.076$ and 0.048 for patients with hypertension with mental problem, cancer, vision problem, heart problem and diabetes, respectively.

Table 2 (last two columns) also report the effects of hypertension and associated comorbidities on HRQoL using the two-part model. In the first part of the model (logistic regression), utility decreased dramatically for most sociodemographic characteristics compared with that in the OLS and Tobit models. Respondents living in an institution were approximately $75 \%$ less likely to report full health, women were $45 \%$ less likely to report full health, and respondents aged $\geq 65$ years were $37 \%$ less likely to report health. For the comorbidity part, respondents who were coliving with mental problem were $10 \%$ less likely to report full health, followed by those with vision problem, heart problem and diabetes. In the second part of the model (truncated OLS), nearly all the sociodemographic characteristics, except for age, exhibited a significant relationship with HRQoL. The pattern in the magnitude of utility for comorbidities was slightly lower than those in the OLS and Tobit models. Respondents with physical problem presented a 0.294 decrease in utility, followed by respondents with mental problem (0.109), cancer $(0.066)$, heart disease $(0.042)$ and diabetes $(0.023)$.

Figure 3 displays the mean utility of patients with hypertension who reported having comorbidities. Among them, respondents with diabetes had the highest utility of 0.83 , followed by those with heart disease, hearing
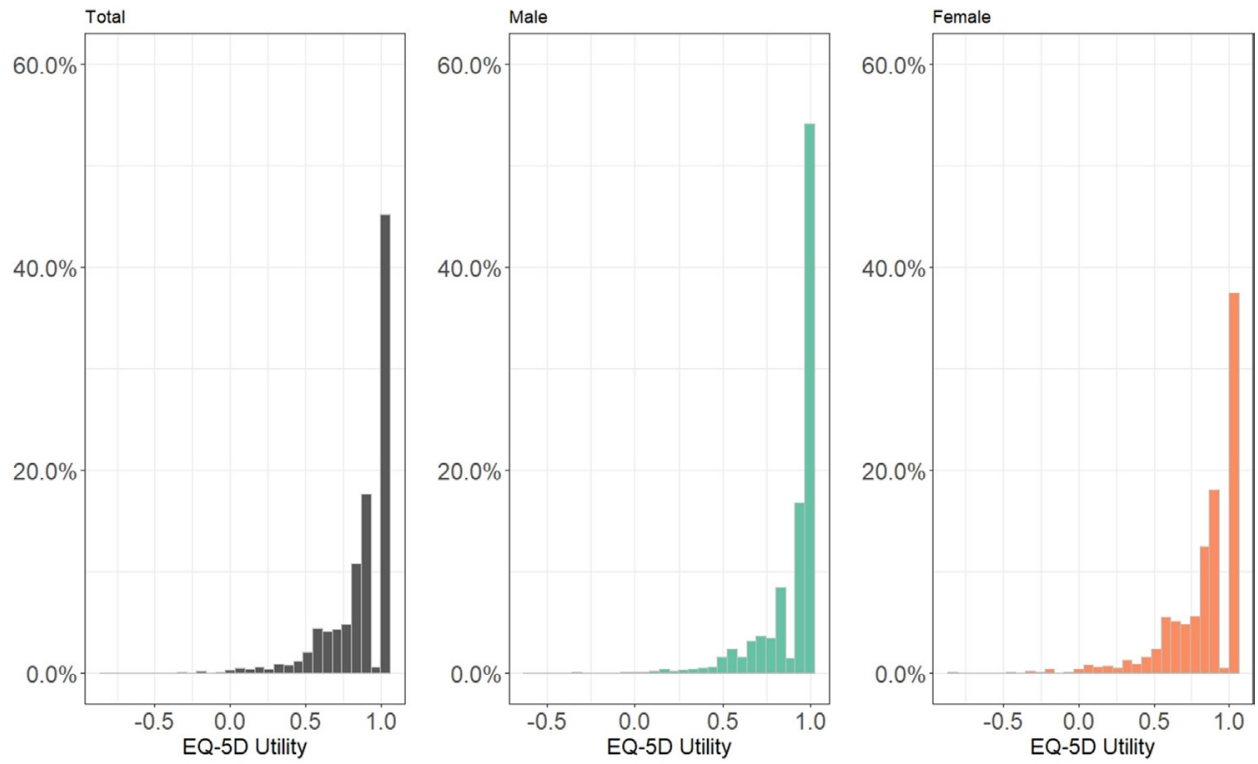

Figure 2 Distribution of EuroQol 5-dimensions utility in the total sample and by sex. 


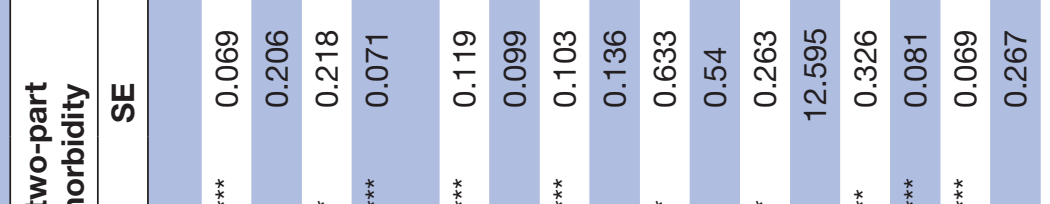

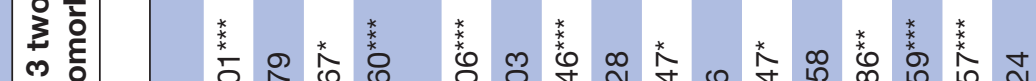

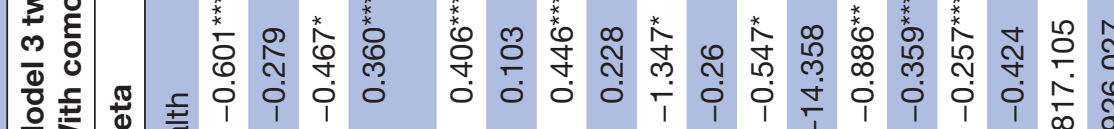

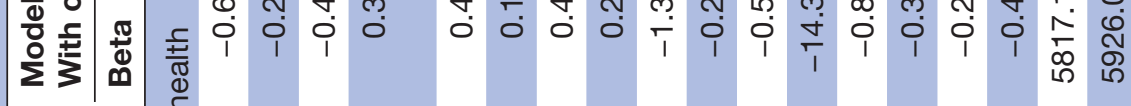

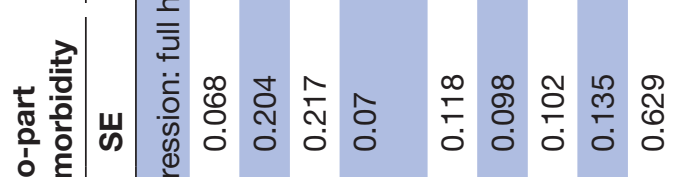

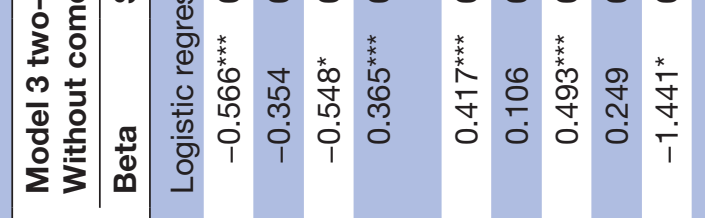

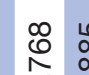

闵 文 $\frac{\sqrt{0}}{\frac{1}{10}}$

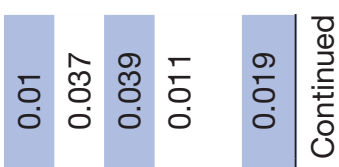

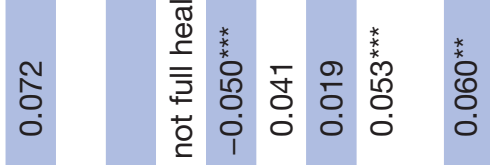

ठํำ

\%

岁

Oे *

오
ஜ
0

ग.

帝

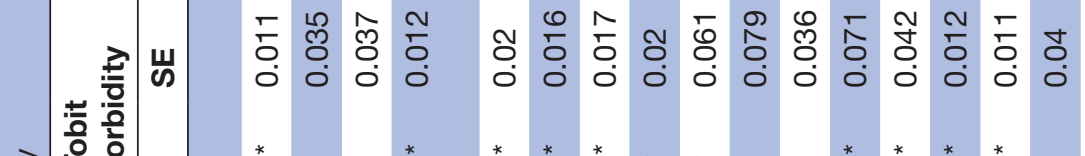

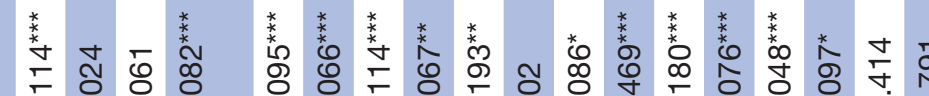

ص

范

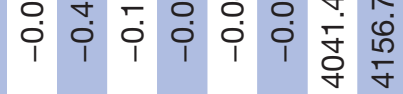

$\frac{5}{5}$

옴

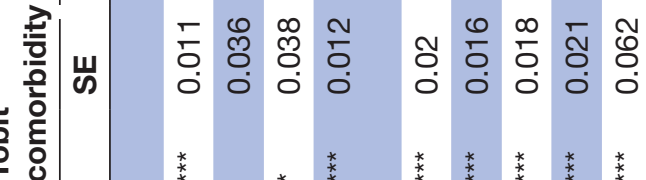

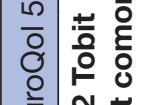

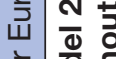

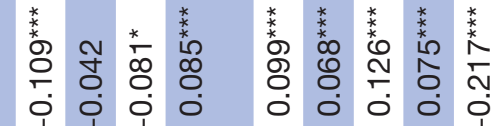

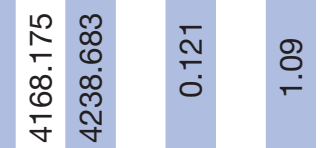

$\frac{\infty}{0} \sum \dot{\Sigma}$

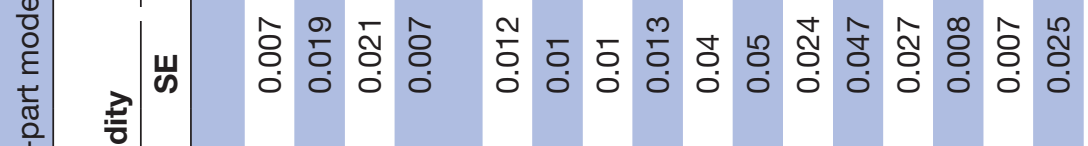

究

等

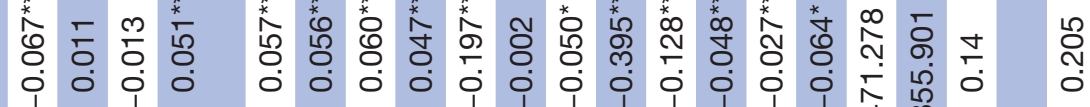

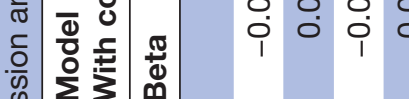

क्र

竞

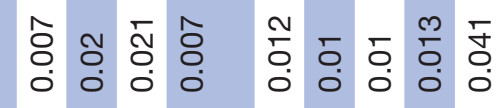

응

की

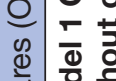

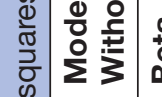

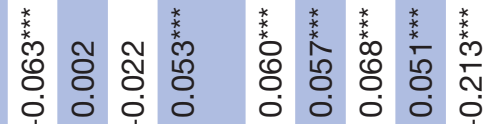

กิ กับ

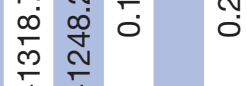

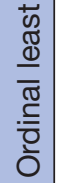

高

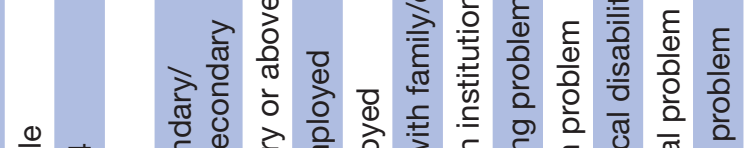

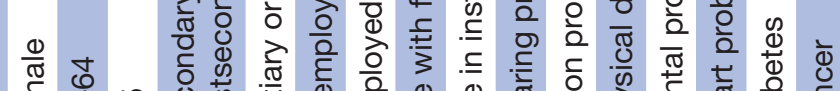

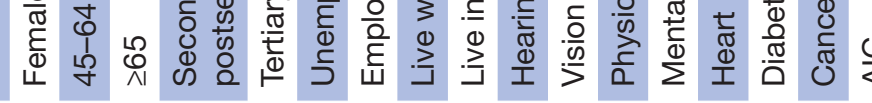

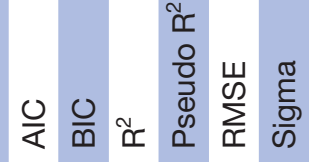



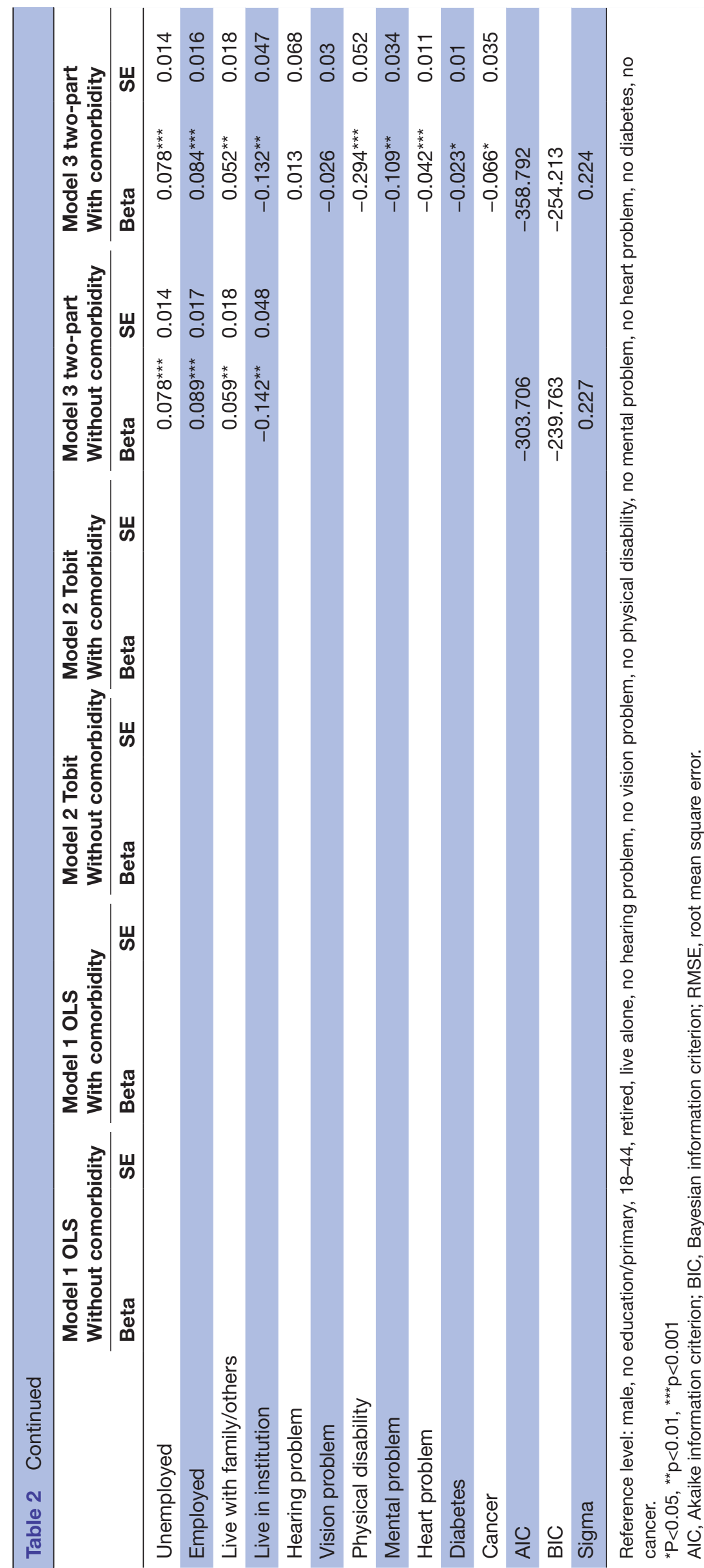


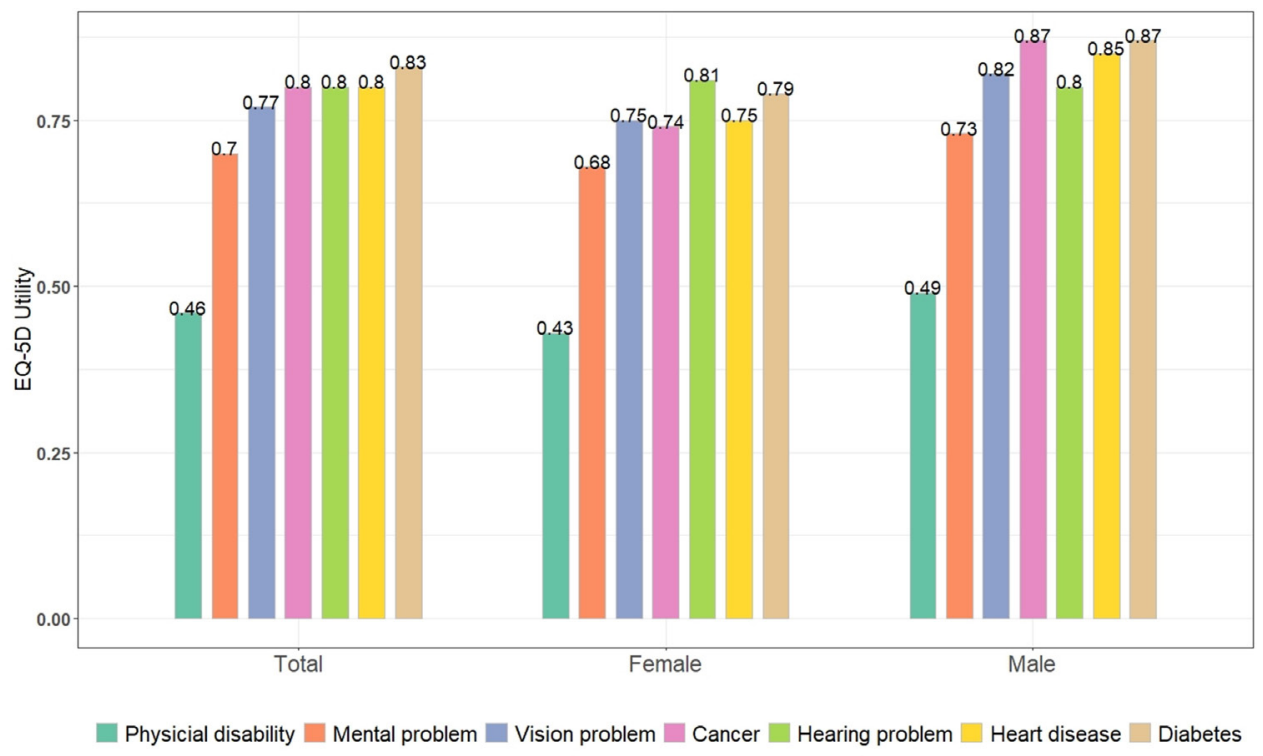

Figure 3 Mean value of EuroQol 5-dimensions (EQ-5D) utility by comorbidities.

problem and cancer. Physical disability scored the lowest with an average of 0.46 .

\section{DISCUSSION}

We conducted this study to explore the effects of hypertension and related comorbidities on HRQoL adjusted by sociodemographic characteristics in the HK population. The results were based on the application of three types of models using EQ-5D-5L HK version. In summary, our models presented the following results. (1) Respondents who were women, elderly, with low educational attainment, live in an institution or retired tended to exhibit low HRQoL. (2) The comorbidity of patients with hypertension associated with other chronic conditions exerted greater effect on decreased HRQoL, particularly for comorbidities with physical disability, mental problem, vision problem, heart problem and diabetes.

In our study, the mean EQ-5D utility for patients with hypertension was 0.85 (mean $=0.92$ for HK general population ${ }^{20}$ ) and ranged from -0.864 to 1.0 . The mean utility was slightly higher than that reported in Japan, ${ }^{25}$ but lower than that reported in South Korea, ${ }^{8}$ Singapore, ${ }^{26}$ Mainland China ${ }^{6}$ and the UK. ${ }^{27}$ This result can be explained by the age-related demographics of our sample. Hypertension is an age-related disease that likely affects older adults. ${ }^{1128}$ In our sample, $60 \%$ of the respondents were reported to be $\geq 65$ years old. This finding may be the reason why hypertension exerts greater negative effect on the HRQoL of HK people. Zahran et al and Cutler et al also reported similar findings in their studies. ${ }^{1029}$ Moreover, the finding of the current study that women exhibited lower utility than men has also been confirmed by studies conducted in China and South Korea and Brazil. ${ }^{6830}$ In Singapore, however, utility is equal for men and women. ${ }^{26}$ By contrast, men reported higher utility than women in Japan. ${ }^{25}$
The prevalence of comorbidity among individuals with hypertension is more common than those without it. ${ }^{31}$ In China, although the comorbidity of hypertension has been extensively studied, two problems remain. First, most research on comorbidity related to hypertension use data from public census or medical survey; the former focuses on facts about the general population, and the latter is constrained by a limited sample size. ${ }^{32}$ Second, EQ-5D-5L is rarely used to evaluate the HRQoL of patients with hypertension in HK. ${ }^{4}$ In this study, we focused on the individual level but also collected information from a large population-based sample to evaluate the effects of the seven types of comorbidity related to hypertension on HRQoL using EQ-5D-5L. Among the seven types, the comorbidity of physical disability with hypertension exhibited the greatest effect on decreased HRQoL, and the magnitude of its effect was at least twice higher than the effects of other comorbidities. Balzi et $a l$ found that older adults with hypertension experience accelerated declines in physical function and increased incidence of disability. ${ }^{9}$ Hajjar et al determined that among nearly 1000 older adults in the USA, high SBP increases the risk of developing new disabilities. ${ }^{33}$ McDaid et al reported that the population aged 50 years and lives with multimorbidity faces the highest risk of disability and poor quality of life. ${ }^{34}$ In HK, studies that discussed the comorbidity of hypertension with physical disability from the perspective of HRQoL are highly limited. Our study can serve as a starting point to facilitate further assessment of the clinical or systematic mechanism behind this comorbidity. Moreover, Hay asserted that sex difference should be considered in the physical changes experience by people who live with hypertension. ${ }^{35}$ Further investigations must be performed to determine whether sex difference influences physical disability to affect the HRQoL of patients with hypertension. We found that pain linked 
to hypertension was closer than the other dimensions of EQ-5D. In the top 10 selected health states of the EQ-5D descriptive system, 6 presented a negative change in the PD dimension. Similar findings were reported in a study conducted in Mainland China, where the PD dimension is the most prominent across the five dimensions in both genders. ${ }^{36}$ Other studies also presented findings that are comparable with ours in both Asian and Western countries ${ }^{826277}$ using EQ-5D-5L and EQ-5D-3L. Potential clinical implications are apparent.

In the current study, we first provided empirical evidence in HK regarding the difference of HRQoL on patients with hypertension between those living with their families and those who do not. Many previous studies have confirmed our findings that family support is essential for the patient to control the hypertension. Ojo et al indicated there is a positive relationship between blood pressure control and family support. ${ }^{38}$ Prazeres and Santiago pointed out that multimorbid patients from dysfunctional families reported worse physical and mental health. ${ }^{39} \mathrm{Xu}$ et al also stated that a poor relationship with family results in a poor state of physical functioning, physical role, social function and emotional role. ${ }^{40}$

Our study has several implications. First, this work is the first to use EQ-5D-5L HK Chinese version to evaluate the HRQoL of people with hypertension and related comorbidities. The results may be used as baselines for HK's hypertensive population in evaluating the effect of policies or clinical interventions to improve the outcome of hypertension care. Second, the EQ-5D utility generated in this study was calculated using the formula developed on the basis of local culture and setting; thus, the bias of using a value set from other countries ${ }^{41}$ or instruments developed based on other culture or settings ${ }^{42}$ is potentially reduced. Third, more than 4500 patients with hypertension successfully participated in the survey and completed the questionnaire, making this work one of the largest population-based surveys in HK to evaluate the HRQoL of patients with hypertension. Fourth, hypertension is the most commonly cited chronic conditions in HK among all non-communicable diseases (NCDs). Studying the effect of hypertension on HRQoL may provide important information to policy-makers in planning a reform for NCD care. Lastly, the adoption of EQ-5D-5L, which is the most widely used measure to assess HRQoL, enabled us to understand and control hypertension based on an international perspective.

Furthermore, we applied three types of regression models to estimate the effect of hypertension and related comorbidities on HRQoL adjusted by the respondents' sociodemographic characteristics. We also presented and compared the performance of each model. The OLS model achieved the best performance in estimation, but it disregarded the bounded nature of EQ-5D utility. ${ }^{22}$ The Tobit model provided reasonable estimation, and the pattern of efficiency was similar to that in the OLS model. The two-part model was flexible and simple, and it provided two independent parts for estimation. However, as Wu et al indicated, two-part models may provide contradicting results and bias when data regarding the full health and non-full health of patients are independently assessed. In this study, cancer showed no significant effect on HRQoL in the logistic model, but it was significant in the truncated OLS model of the two-part model. We should explain the results of the two-part model with caution. ${ }^{22}$

Our study has several limitations. First, the distribution of patient characteristics was skewed towards elderly people. Second, we did not differentiate hypertension in terms of types, severities or stages. Such differences may have varying effects on HRQoL. Third, we only considered seven types of comorbidity in the estimation; thus, additional chronic conditions and multimorbidities $(\geq 3$ chronic conditions) should be considered. Lastly, the nature of a cross-sectional study could have introduced certain biases on the generalisation of our findings.

\section{CONCLUSION}

This study applied the EQ-5D-5L HK Chinese version to a large population-based sample of patients with hypertension in HK. Respondents with hypertension were men, with a high education, fully employed and living with families have better HRQoL. Respondents who living with comorbidity were prone to report lower HRQoL, particularly those with physical disability and mental problem. However, respondents who living with hearing problem did not significantly decrease HRQoL of patients with hypertension. This study provides detailed information of the implications for healthcare providers to gain a more complete picture of health of their patients with hypertension. In the future, additional study using longitudinal data set is required to observe whether finding remains over time and if so the causality relationship between hypertension and comorbidity and HRQoL could be established.

Contributors All authors jointly conceived of the research. RHX prepared the manuscript and completed the statistical analysis. All authors have full access to all the data in the study and review the manuscript.

Funding The authors have not declared a specific grant for this research from any funding agency in the public, commercial or not-for-profit sectors.

Competing interests None declared.

Patient consent for publication Not required.

Ethics approval The ethical approval of original PESS was obtained from the Chinese University of HK and the New Territory East Cluster Clinical Research Ethics Committee.

Provenance and peer review Not commissioned; externally peer reviewed.

Data availability statement № additional data are available.

Open access This is an open access article distributed in accordance with the Creative Commons Attribution Non Commercial (CC BY-NC 4.0) license, which permits others to distribute, remix, adapt, build upon this work non-commercially, and license their derivative works on different terms, provided the original work is properly cited, appropriate credit is given, any changes made indicated, and the use is non-commercial. See: http://creativecommons.org/licenses/by-nc/4.0/.

ORCID iD

Richard Huan Xu http://orcid.org/0000-0002-4720-5172 


\section{REFERENCES}

1. RodrigoF, JoseM, GiulliardOC, et al. Hypertension and aging. J Clin Hypertens 2013;15.

2. World Health Organization. A global brief on hypertension: World health day 2013.

3. The Department of Health. Hong Kong reference framework for hypertension care for adults in primary care settings, 2018.

4. Xu RH, Cheung AWL, Wong EL-Y. Examining the health-related quality of life using EQ-5D-5L in patients with four kinds of chronic diseases from specialist outpatient clinics in Hong Kong SAR, China. Patient Prefer Adherence 2017:11:1565-72.

5. Alonso J, Ferrer M, Gandek B, et al. Health-Related quality of life associated with chronic conditions in eight countries: results from the International quality of life assessment (IQOLA) project. Qual Life Res 2004;13:283-98.

6. Zhang Y, Zhou Z, Gao J, et al. Health-related quality of life and its influencing factors for patients with hypertension: evidence from the urban and rural areas of Shaanxi Province, China. BMC Health Serv Res 2016;16:277.

7. Wang J-G, Li Y. Characteristics of hypertension in Chinese and their relevance for the choice of antihypertensive drugs. Diabetes Metab Res Rev 2012;28(Suppl 2):67-72.

8. Lee MH, So ES. Impact of hypertension-related comorbidity on health-related quality of life: a population-based survey in South Korea. Asia Pac J Public Health 2012;24:753-63.

9. Balzi D, Lauretani F, Barchielli A, et al. Risk factors for disability in older persons over 3-year follow-up. Age Ageing 2010;39:92-8.

10. Cutler JA, Sorlie PD, Wolz M, et al. Trends in hypertension prevalence, awareness, treatment, and control rates in United States adults between 1988-1994 and 1999-2004. Hypertension 2008;52:818-27.

11. Pinto E. Blood pressure and ageing. Postgrad Med J 2007;83:109-14

12. Trevisol DJ, Moreira LB, Kerkhoff A, et al. Health-related quality of life and hypertension: a systematic review and meta-analysis of observational studies. J Hypertens 2011;29:179-88.

13. Reenen Mvan, Janssen B, et al. EQ-5D-5L user guide - basic information on how to use the EQ-5D-5L instrument. Version 5.1, 2015.

14. McClure NS, Sayah FA, Ohinmaa A, et al. Minimally important difference of the EQ-5D-5L index score in adults with type 2 diabetes. Value Health 2018;21:1090-7.

15. Huang W, Yang J, Liu Y, et al. Assessing health-related quality of life of patients with colorectal cancer using EQ-5D-5L: a cross-sectional study in Heilongjiang of China. BMJ Open 2018;8:e022711.

16. Dyer MTD, Goldsmith KA, Sharples LS, et al. A review of health utilities using the EQ-5D in studies of cardiovascular disease. Health Qual Life Outcomes 2010;8:13.

17. Ascef BdeO, Haddad JPA, Álvares J, et al. Health-Related quality of life of patients of Brazilian primary health care. Rev Saude Publica 2017;51(suppl 2):22s.

18. Xu RH, Wong EL. Involvement in shared decision-making for patients in public specialist outpatient clinics in Hong Kong. Patient Prefer Adherence 2017;11:505-12.

19. Wang L, Palmer AJ, Cocker F, et al. Multimorbidity and healthrelated quality of life (HRQOL) in a nationally representative population sample: implications of count versus cluster method for defining multimorbidity on HRQoL. Health Qual Life Outcomes 2017;15:7.

20. Wong ELY, Ramos-Goñi JM, Cheung AWL, et al. Assessing the use of a feedback module to model EQ-5D-5L health states values in Hong Kong. Patient 2018;11:235-47.

21. Wilcox RR. Introduction to robust estimation and hypothesis testing. 3rd edn. Elsevier Science, 2011.

22. Wu M, Brazier JE, Kearns B, et al. Examining the impact of 11 long-standing health conditions on health-related quality of life using the EQ-5D in a general population sample. Eur J Health Econ 2015;16:141-51.

23. Starkie HJ, Briggs $\mathrm{AH}$, Chambers MG, et al. Predicting EQ-5D values using the SGRQ. Value Health 2011;14:354-60.

24. Young TA, Mukuria C, Rowen D, et al. Mapping functions in healthrelated quality of life: mapping from two cancer-specific healthrelated quality-of-life instruments to EQ-5D-3L. Med Decis Making 2015;35:912-26.

25. Fujikawa A, Suzue T, Jitsunari F, et al. Evaluation of health-related quality of life using EQ-5D in Takamatsu, Japan. Environ Health Prev Med 2011;16:25-35.

26. Abdin E, Subramaniam M, Vaingankar JA, et al. Population norms for the EQ-5D index scores using Singapore preference weights. Qual Life Res 2015;24:1545-53.

27. Marques da Silva P, Haag U, Guest JF, et al. Health-related quality of life impact of a triple combination of olmesartan medoxomil, amlodipine besylate and hydrochlorotiazide in subjects with hypertension. Health Qual Life Outcomes 2015;13:24.

28. Anderson GH. Effect of age on hypertension: analysis of over 4,800 referred hypertensive patients. Saudi J Kidney Dis Transp/ 1999;10:286-97.

29. Zahran HS, Kobau R, Moriarty DG, et al. Health-related quality of life surveillance-United States, 1993-2002. MMWR Surveill Summ 2005;54.

30. Carvalho MVde, Siqueira LB, Sousa ALL, et al. The influence of hypertension on quality of life. Arq Bras Cardiol 2013;100:164-74.

31. Noh J, Kim HC, Shin A, et al. Prevalence of comorbidity among people with hypertension: the Korea National health and nutrition examination survey 2007-2013. Korean Circ J 2016;46:672.

32. Liu J, Ma J, Wang J, et al. Comorbidity analysis according to sex and age in hypertension patients in China. Int J Med Sci 2016;13:99-107.

33. Hajjar I, Lackland DT, Cupples LA, et al. Association between concurrent and remote blood pressure and disability in older adults. Hypertension 2007;50:1026-32.

34. McDaid O, Hanly MJ, Richardson K, et al. The effect of multiple chronic conditions on self-rated health, disability and quality of life among the older populations of Northern Ireland and the Republic of ireland: a comparison of two nationally representative cross-sectional surveys. BMJ Open 2013;3:e002571.

35. Hay M. Sex, the brain and hypertension: brain oestrogen receptors and high blood pressure risk factors. Clin Sci 2016;130:9-18.

36. Liang Z, Zhang T, Lin T, et al. Health-related quality of life among rural men and women with hypertension: assessment by the EQ-5D5L in Jiangsu, China. Qual Life Res 2019;28:2069-80.

37. N'Goran AA, Déruaz-Luyet A, Haller DM, et al. Comparing the self-perceived quality of life of multimorbid patients and the general population using the EQ-5D-3L. PLoS One 2017;12:e0188499.

38. Ojo OS, Malomo SO, Sogunle PT. Blood pressure (BP) control and perceived family support in patients with essential hypertension seen at a primary care clinic in Western Nigeria. J Family Med Prim Care 2016;5.

39. Prazeres F, Santiago L. Relationship between health-related quality of life, perceived family support and unmet health needs in adult patients with multimorbidity attending primary care in Portugal: a multicentre cross-sectional study (Survey). Health Qual Life Outcomes 2016;14:156.

40. Xu X, Rao Y, Shi Z, et al. Hypertension impact on health-related quality of life: a cross-sectional survey among middle-aged adults in Chongqing, China. (Research Article) (Survey). Int J Hypertens 2016;2016:7404957

41. Cheung PWH, Wong CKH, Cheung JPY. Differential psychometric properties of EuroQoL 5-Dimension 5-level and shortform 6-dimension utility measures in low back pain. Spine 2019;44:E679-86.

42. Lam ETP, Lam CLK, Fong DYT, et al. Is the SF-12 version 2 health survey a valid and equivalent substitute for the SF-36 version 2 health survey for the Chinese? J Eval Clin Pract 2013;19:200-8. 